\title{
Jefferson。
}

\section{The Significance of Percutaneous Aspiration of the Zygapophysial Facet Joint Synovial Cyst: A Case Series}

\section{S Kamal Fetouh', Nicholas Kaffi2 , , Linqiu Zhou ${ }^{1,2}$}

1 Department of Rehabilitation Medicine, Thomas Jefferson University, Philadelphia, PA; 2 Spinal Surgery \& Pain Management, Woodbury, NJ

\begin{tabular}{l} 
INTRODUCTION \\
Synovial cysts (SC) of the zygapophysial \\
(ZP) joints are a source of nerve root \\
compression. An uncommon cause of \\
lumbar radiculopathy., the incidence of ZP \\
SC as a cause of radicular pain based on \\
MRI has been suggested to be $0.65 \%$. \\
They are most common between the \\
fourth and fifth lumbar vertebrae (68\%) \\
with the majority occurring on the medial \\
aspect of ZP joints within the posterolateral \\
aspect of the spinal canal at $2-5$ 'clock on \\
the left (L) or $7-10$ o'clock on the right (R) \\
positions \\
They are thought to arise in association \\
with degenerative disease of the spine. \\
Different interventional procedures are in \\
use for decompression including invasive \\
surgical decompression. \\
Percutaneous aspiration with fluoroscopic \\
guidance, a non-surgical interventional \\
technique, may be as efficacious if not \\
better than the aforementioned. \\
MRI scans facilitate assessment of cyst \\
orientation for proper needle positioning in \\
addition to the size of the cyst and severity \\
of spinal compression before and after \\
procedure. \\
In order to clarify the role of percutaneous \\
aspiration, we analyzed factors influencing \\
the outcome in a series of 4 patients. \\
\hline
\end{tabular}

\section{CASE REPORT}

As per MRI, 3 PTs ZP SC were located at the $L$ medial L4-5 level. 2 PTs were male and 1 female. The 4 th $P T$ was a male with a $L$ medial ZP SC at L5-S1 leve

All Pts failed a series of 3 epidural steroid injections for their radicular back pain prior to percutaneous aspiration of the ZP SC under fluoroscopy.

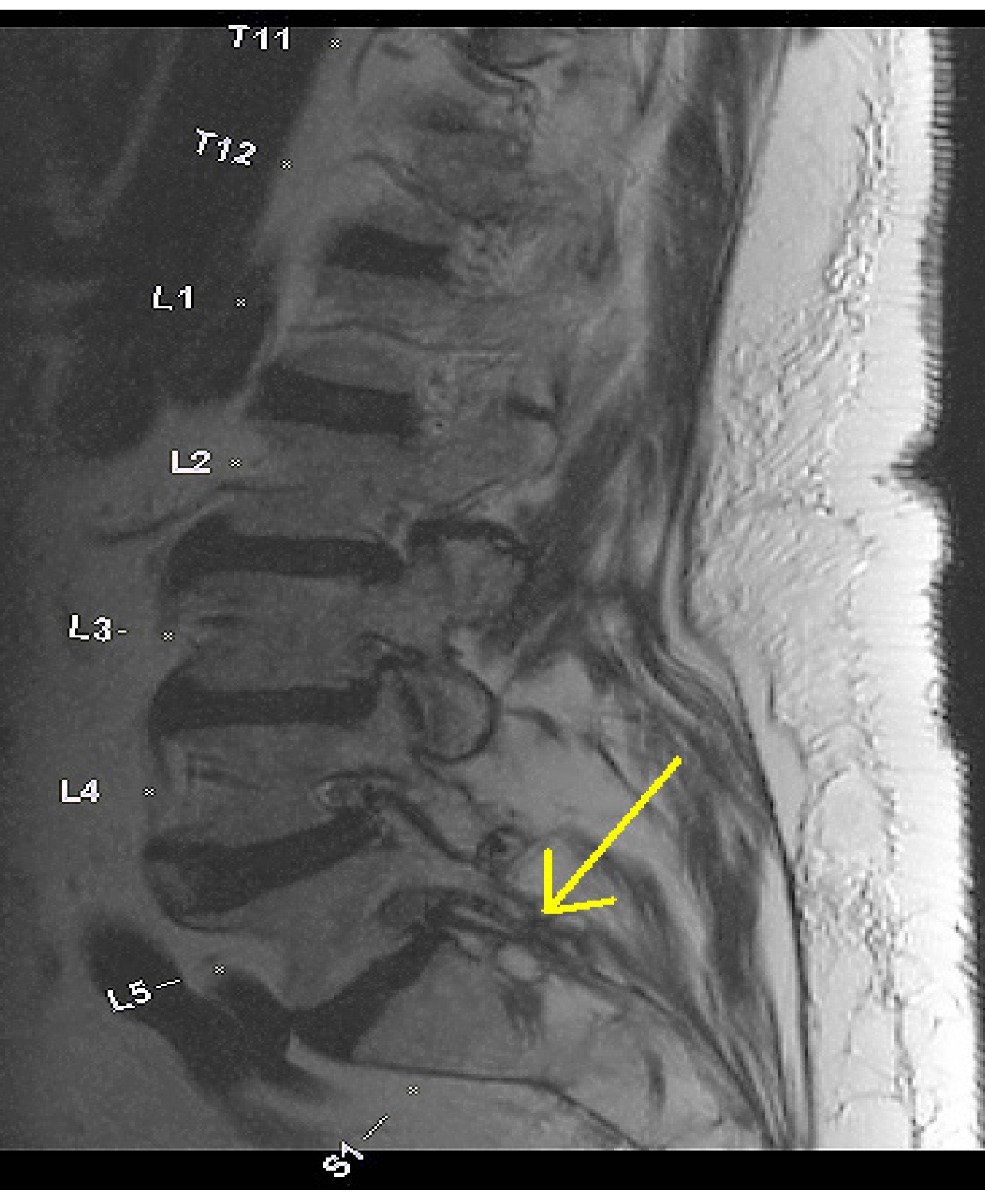

Fig. 1 Sagittal View Prior to Aspiration

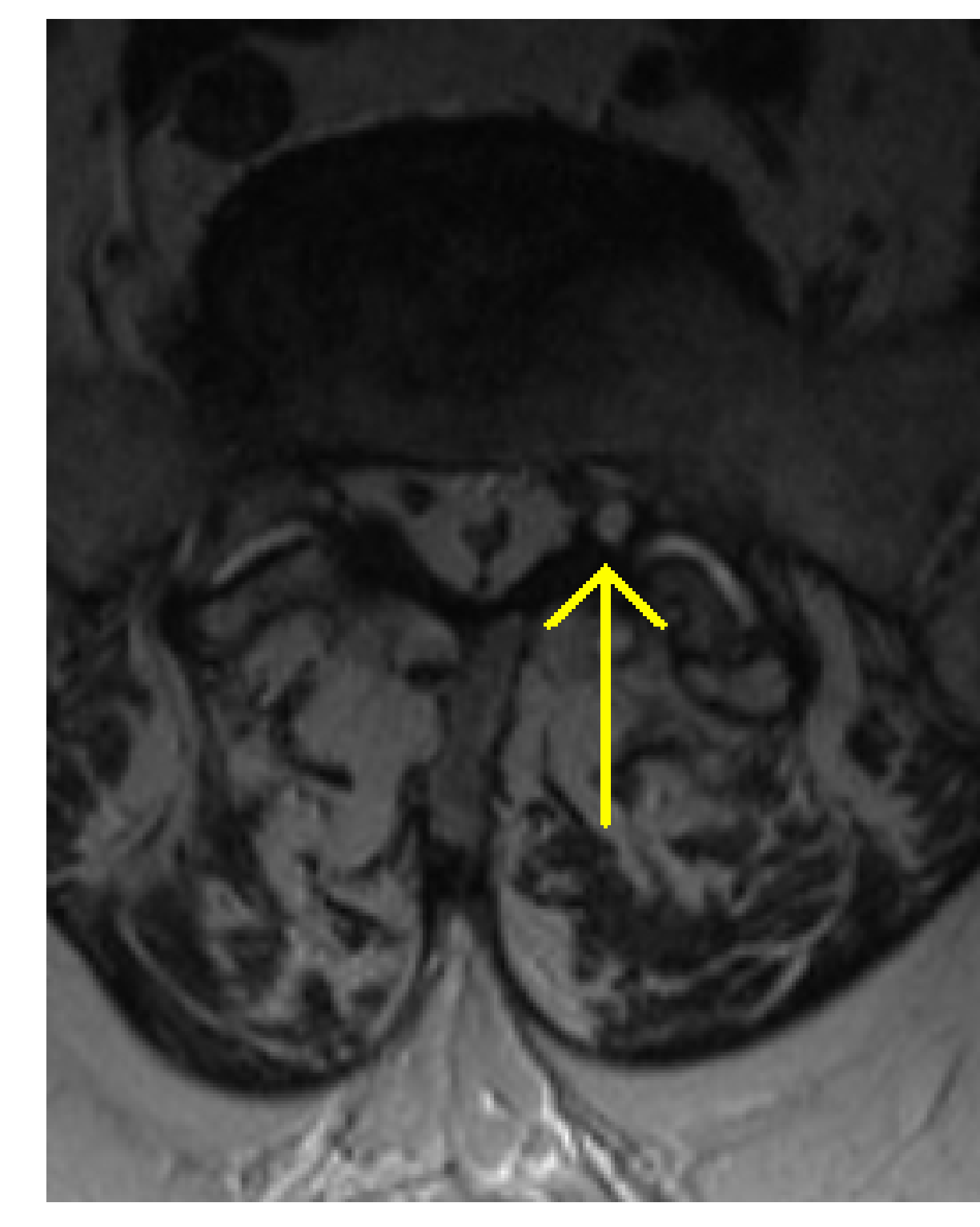

Fig. 2 Axial View Prior to Aspiration

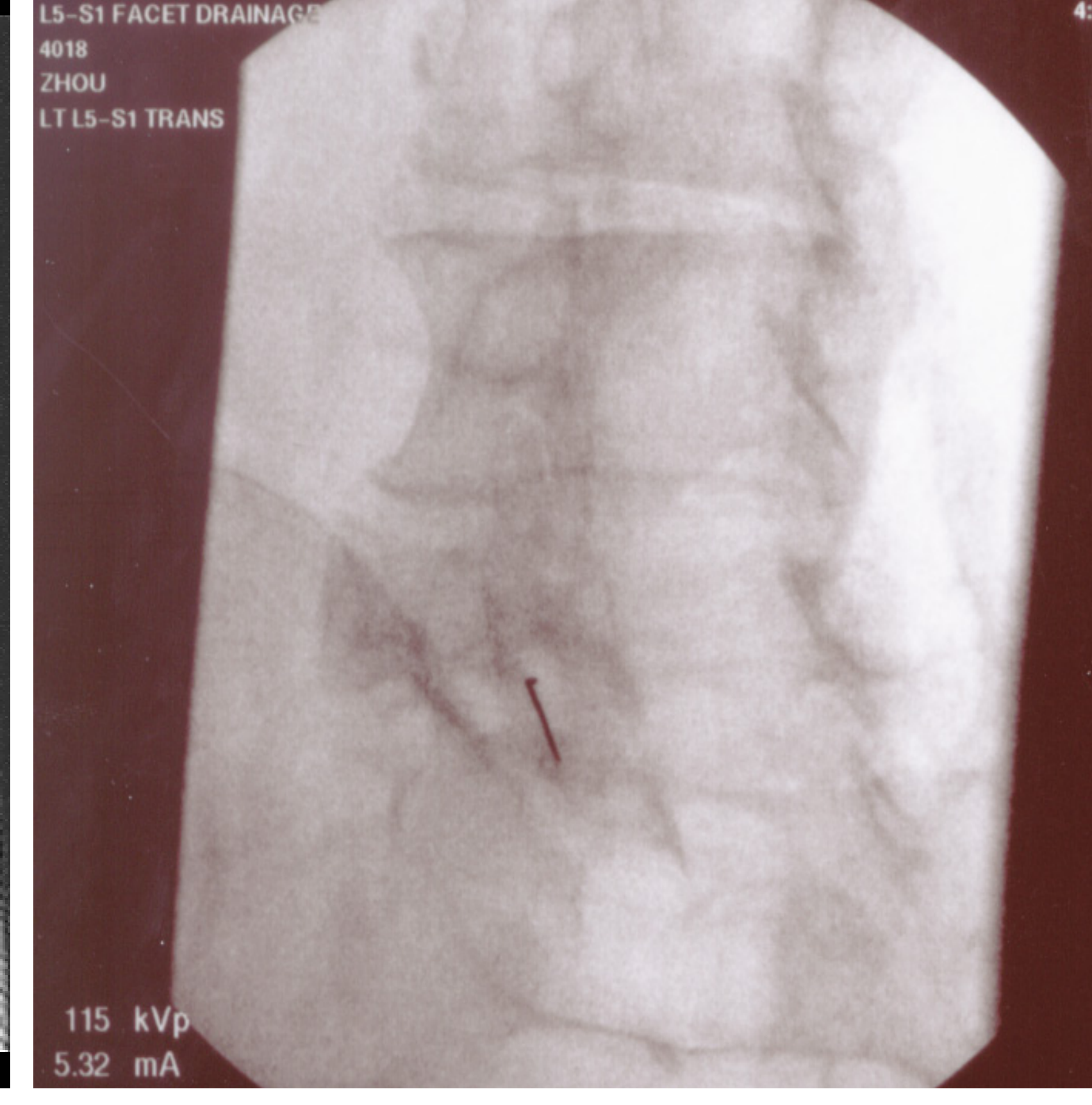

Fig. 3 Fluoroscopic Guided Aspiration

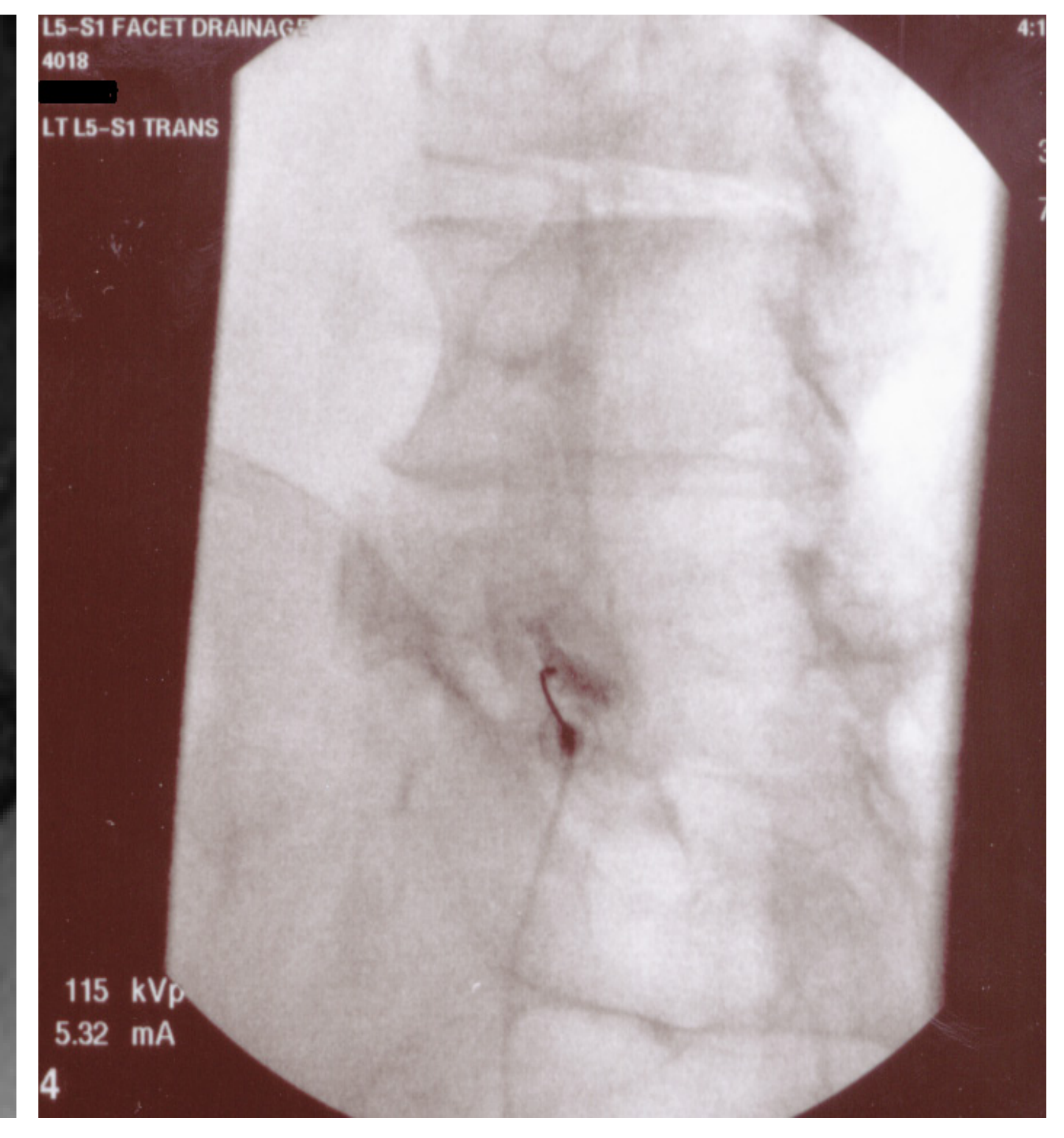

Fig. 4 Contrast Dye Revealing the Cyst under Fluoroscopy

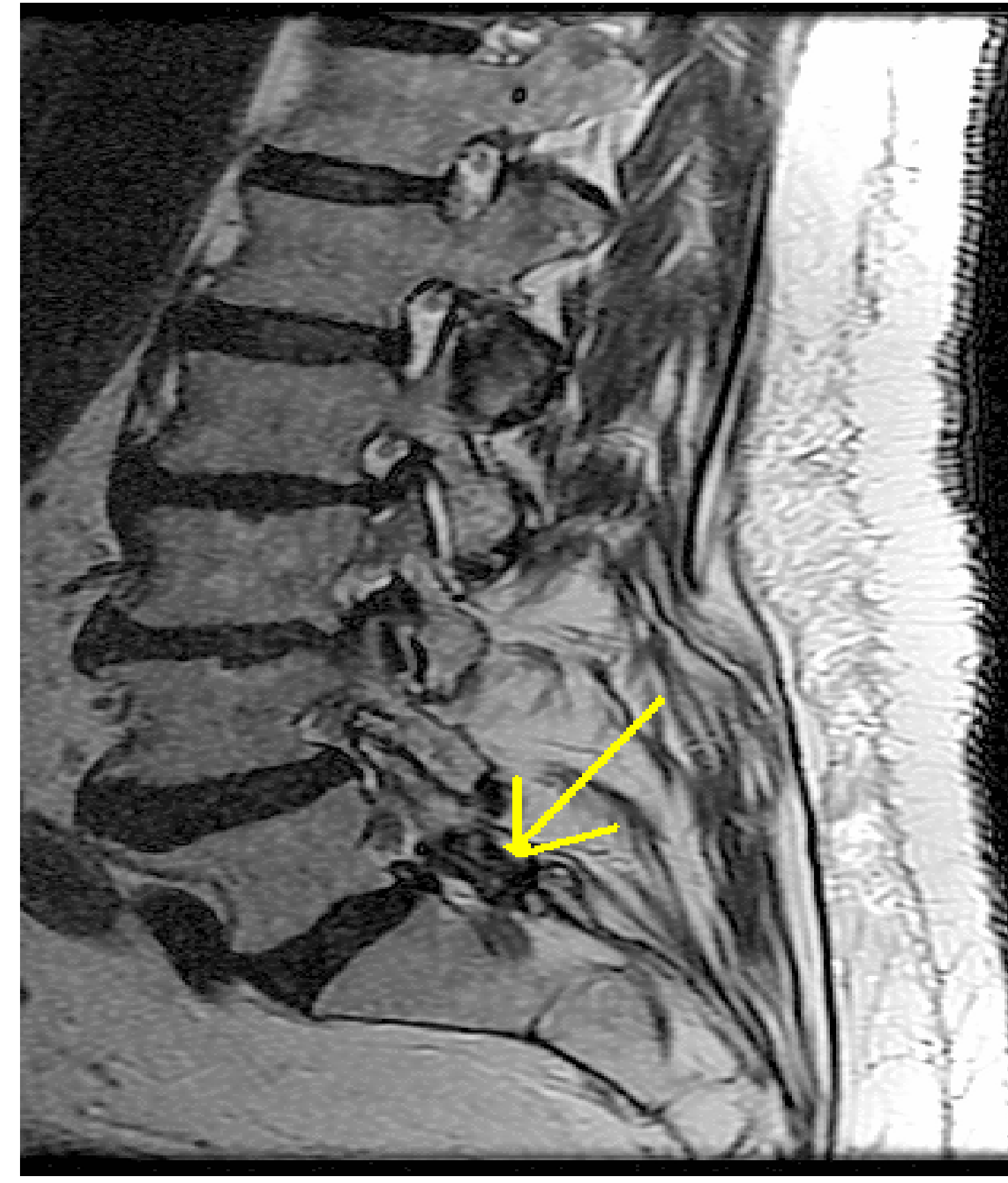

Fig. 5 Sagittal View Post Aspiration and Decompression

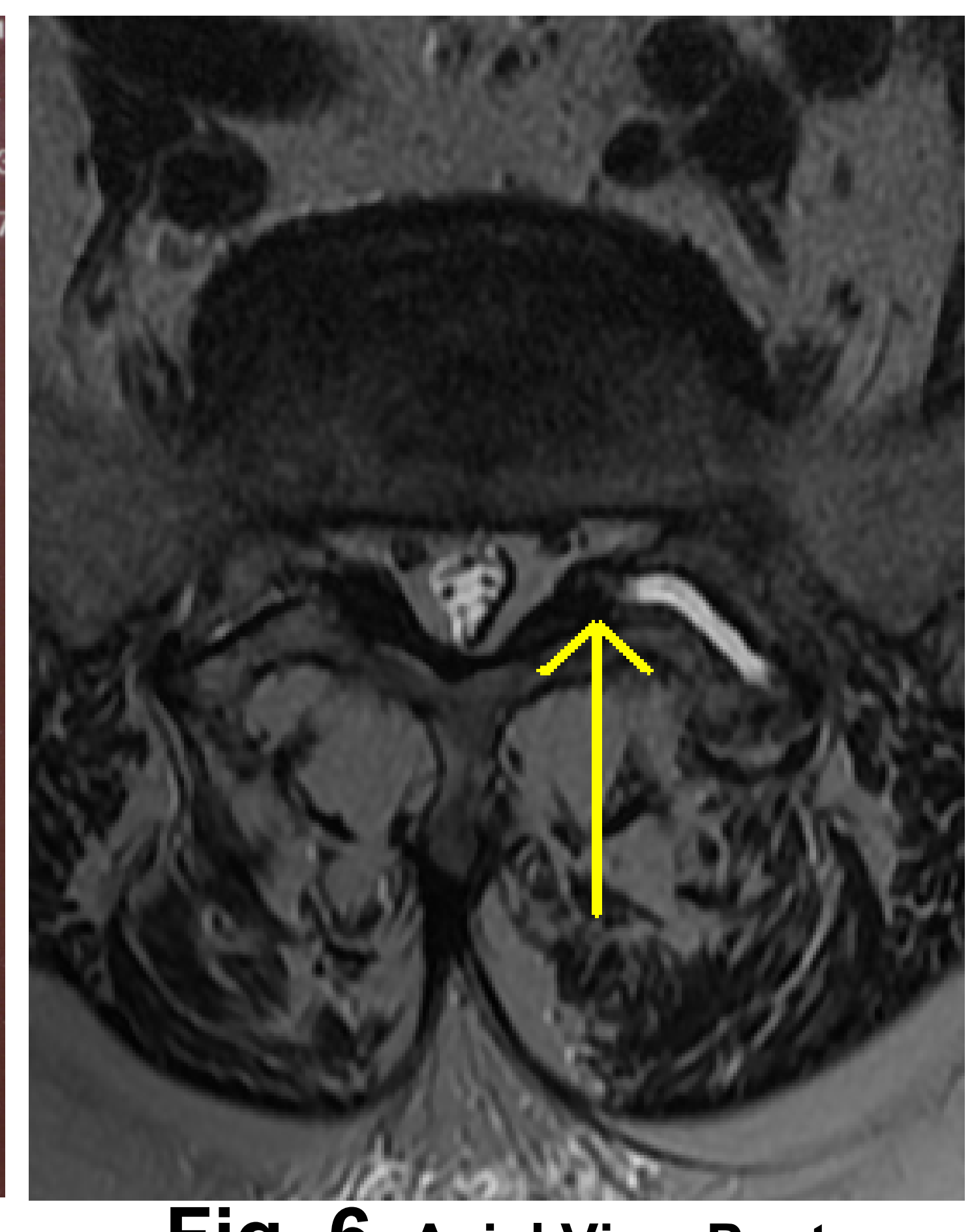

Fig. 6 Axial View Post Aspiration and Decompression

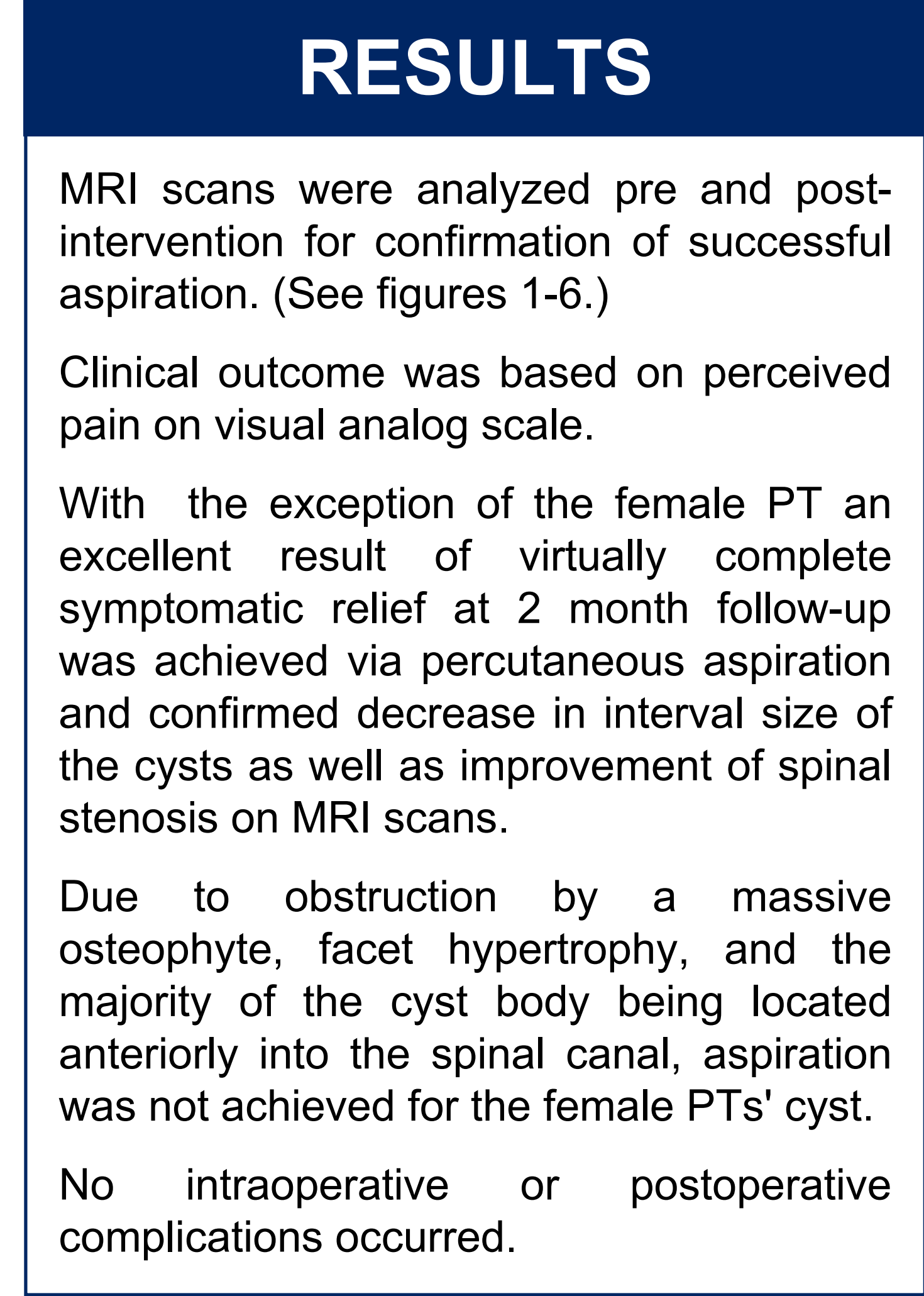

\section{CONCLUSION}

Cysts that are clearly associated with threat of progressive neurological loss or intractable, unremitting symptoms should be released by a decompression procedure.

In our case series, percutaneous aspiration has been successfully used for 3 of 4 PTs with marked decrease in size of cyst on imaging and clinical improvement. Obstructing pathology and ultimately the location of the cyst can be major factors in determining the success of the procedure.

Our study demonstrated that a minimally invasive aspiration of a ZP SC can often achieve clinical improvement.

This may save the PT from undergoing an invasive surgical decompression. 